\title{
Original Article \\ Conventional Echocardiography Versus Tissue Doppler in Children with Primary Nephrotic Syndrome.
}

\author{
Ashraf S. Kamel ${ }^{1}$, Sara I. AboElnour ${ }^{1}$, Mohamed A. El-Gamasy ${ }^{2}$, Marwa M. Ragaey ${ }^{1}$ \\ 1- Department of Pediatrics, Faculty of Medicine, Fayoum University, Fayoum, Egypt. \\ 2- Department of Pediatrics, Faculty of Medicine, Tanta University, Tanta, Egypt.
}

\begin{abstract}
Introduction: Cardiac affection in patients with nephrotic syndrome (NS) may be attributable to malnutrition and activated inflammatory state. Myocardial performance index (MPI) is more sensitive than the classic echocardiographic parameters, particularly in showing subclinical myocardial function compromise.

Aim of the study: Tto compare the findings of conventional echocardiography and tissue Doppler imaging in children with NS.

Methods: This study was conducted at the outpatient Nephrology Clinic and the Cardiology Unit of Pediatric Department at Fayoum University Hospital. It included 30 patients with primary nephrotic syndrome (PNS) compared with 30 apparently healthy subjects, age- and gender-matched to the diseased group. Participants were subjected to full history taking, thorough clinical examination, anthropometric measurements, and laboratory studies. Conventional echocardiography and tissue Doppler imaging were used for evaluation of ventricular hemodynamics.

Results: Conventional echocardiography did not reveal any significant differences between the patients and control subjects. Tissue Doppler imaging showed significant differences in some parameters. The right ventricular (RV) and left ventricular (LV) MPI were high in $30 \%$ and $20 \%$ of PNS patients, respectively. Both LV and RV dysfunctions were significantly associated with longer duration of disease and treatment as well as higher percentage of relapse, steroid resistance, and intake of immunosuppressives.

Conclusion: Both LV and RV dysfunctions may occur in children with PNS. Both conditions were significantly associated with disease duration, treatment duration, relapse, steroid resistance, and administration of immunosuppressive drugs. Tissue Doppler echocardiography is recommended for follow-up of NS patients, particularly those with long disease duration and/or prescribed immunosuppressive drugs.
\end{abstract}

Keywords: child; conventional echocardiography; myocardial performance index; nephrotic syndrome; tissue doppler imaging.

Running Title: Conventional echocardiography \& tissue doppler in INS

Corresponding author: Ashraf Sayed Kamel, MD

Department of Pediatrics, Faculty of Medicine, Fayoum University, Fayoum, Egypt;

Postal code: 63514 ;

Email: ashraf.s.kamel22@gmail.com \& ask00@fayoum.edu.eg

Phone: +201225876153

ORCID: 0000-0001-6822-2150

geget: The Journal of the Egyptian Society of Pediatric Nephrology and Transplantation (ESPNT)

geget https://geget.journals.ekb.eg/

Published by ESPNT http://espnt.net/

Cohosted by Egyptian Knowledge Bank https://www.ekb.eg

Copyright 2021. All rights reserved $\subset$ ESPNT ( geget ) 


\section{INTRODUCTION}

Childhood nephrotic syndrome (NS) is one of the most common pediatric kidney diseases, which has an incidence of approximately 1.2 to 16.9 per 100000 children.[1, 2] It is characterized by a triad of proteinuria, edema, and hypoalbuminemia [3]. Nephrotic syndrome may be primary with a disease specific to the kidneys or secondary to congenital infections, diabetes, systemic lupus erythematosus, neoplasia, or certain drug use [4].

There is an increased incidence of heart disease in patients with primary nephrotic syndrome (PNS) compared to the normal population [5]. Several mechanisms were proposed to explain the occurrence of heart disease in these patients. One potential mechanism is the activation of inflammatory reaction and the effect of cytokines on the myocardium $[6,7]$. Massive proteinuria results in hypoalbuminemia, which in turn causes edema. Intestinal edema develops and reduces nutrient absorption, leading to malnutrition and activation of the inflammatory cascade. Various inflammatory mediators (such as TNF- $\alpha$ ) exhibit a negative inotropic effect on the myocardium [8].

The effect of acute afterload elevations was also suggested to result in decreased relaxation rate and increased diastolic intolerance to afterload in children with PNS $[9,10]$.

Another suggested mechanism for the increased incidence of heart disease in PNS patients is the occurrence of coronary artery disease secondary to hypercoagulability and dyslipidemia. Primary nephrotic syndrome is also associated with hypercoagulability and an increased tendency for thromboembolism. The reported incidences of thromboembolic complications ranged from $1.8 \%$ to $6.6 \%$ in children with PNS. Although thromboembolism may occur anywhere, deep vein thrombosis and pulmonary embolism are the most frequently encountered manifestations in the clinical setting and may be correlated with right ventricle (RV) function. [11, 12] In addition, dyslipidemia is probably responsible for endothelial dysfunction in the conduit arteries in patients with nephrosis and could form the basis for the increased risk of cardiovascular disease in these patients $[13,14]$.

Conventional Echocardiography is usually utilized to diagnose myocardial dysfunction. Tissue Doppler imaging is a non-invasive cardiac imaging technique which measures the velocity of the longitudinal motion of the mitral annulus, tricuspid annulus and the basal part of interventricular septum [15]. The aim of the current study was to compare the findings of conventional echocardiography and tissue Doppler imaging in children with NS.

\section{METHODS}

The study was conducted between January 2019 and September 2019 at both the outpatient Nephrology Clinic and Cardiology Unit of Fayoum University Hospital.

This case control study included 30 children and adolescents (age: 4 to 14 years old) diagnosed with PNS and had regular follow-up at the outpatient Nephrology Clinic and Cardiology Unit of our University Hospital in addition to 30 
age- and gender-matched healthy subjects recruited as a control group.

We included cases based on the following criteria: hypoalbuminemia (serum albumin $<2.5 \quad \mathrm{~g} / \mathrm{dl}$ ), hypercholesterolemia (serum cholesterol $>250 \mathrm{mg} / \mathrm{dl}$ ), generalized edema, and heavy proteinuria (urinary protein $>2$ $\mathrm{gm} / 24 \mathrm{~h}$ ). We excluded patients with known congenital or acquired heart diseases and those with pulmonary diseases. In addition, patients who were below 4- or above 14-years-old and those who refused to participate were excluded.

The study participants were subjected to thorough history taking, clinical examination, and laboratory investigations [including complete blood count, liver function tests, blood urea, serum creatinine, total blood cholesterol levels, and albumin/creatinine $(\mathrm{A} / \mathrm{C})$ ratio in a fresh morning urine sample].

Echocardiography was performed using a Vivid 5-color Doppler ultrasound system (General Electric Healthcare, Milwaukee, WI, USA) with transducers of $3.75 \mathrm{MHz}$ or $5 \mathrm{MHz}$, as appropriate for children and adolescents. A complete echocardiographic examination was performed to exclude the possibility of congenital heart disease with great emphasis on right ventricle (RV) dimensions, global function, and left ventricle (LV) internal dimensions with assessment of LV ejection fraction. The recorded images for each patient were stored and analyzed offline with the software blinded to the patients' data. From the standard transthoracic windows, LV end diastolic diameter (LVEDD), LV end systolic diameter (LVESD), LV posterior wall (LVPW), and LV ejection fraction (EF) were measured. Transmitral
E wave velocity (E) and A wave velocity (A) were obtained from the recorded data and were averaged to generate the mean value.

Myocardial performance index (MPI) was calculated by dividing the sum of isovolumic relaxation time (IVRT) and isovolumic contraction time (IVCT) by ejection time (ET) [16, 17].

RV MPI $=($ IVCT + IVRT $) /$ RVET

LV MPI $=($ IVCT + IVRT $) /$ LVET

Then, we compared patients with high and normal MPI of the right ventricle as regards disease characteristics (disease duration, treatment duration, $1^{\text {st }}$ attack or relapse, steroid sensitivity, and the use of immune suppressive drugs).

\section{Ethical considerations:}

This study complies with all the relevant national regulations, institutional policies and in accordance with the tenets of the Helsinki Declaration and has been approved by our Research Ethics Committee. Informed written consent was obtained from the father or the legal guardian of each child after explanation of the aim of the study and its methods. Confidentiality of patients' information was ensured by assigning a code number to each patient known only by the investigators, keeping data collection sheets anonymous(identified by the code number), and preserving the file containing the names and codes safe from intrusion.

\section{Statistical analysis:}

The collected data were organized, tabulated, and statistically analyzed using SPSS software, version 22 (IBM Corp., Armonk, N.Y., USA). Quantitative were expressed as the mean \pm standard deviation. Comparison between any two groups as regards the study variables was 
done using the independent samples $\mathrm{T}$ test. Qualitative data were described as frequencies (number) and percentages; Chi-square test was used as a test of significance. Significance was adopted at $\mathrm{p} \leq 0.05$.

\section{RESULTS}

The demographic characteristics of the study groups are summarized in Table 1. The mean age was $6.5 \pm 3.3$ years for the PNS group and $7.3 \pm 2.4$ years for the control group. The PNS patient group included 19 males and 11 females and the control group included 13 males and 17 females. There were no significant differences between the two groups as regards age $(\mathrm{p}=0.334)$ or gender $(\mathrm{p}=$ 0.121 ).

Findings of echocardiography and tissue Doppler in both study groups are shown in Tables $2 \& 3$, and there was no significance difference between patient and control groups regarding the characteristics of conventional echocardiography $\quad(p>0.05)$ Tissue Doppler showed in the cases groups the presence of significantly higher mean right ventricular IVCT $(\mathrm{p}<0.001)$, higher mean LV IVRT $(p=0.005)$, lower mean LV IVCT $(p=0.032)$ as well as lower mean transmitral $\mathrm{E}(\mathrm{p}=0.003)$ and $\mathrm{A}(\mathrm{p}=$ 0.003 ) wave velocities, compared to the control group. We evaluated right ventricular and left ventricular function by MPI; increased MPI indicates diastolic dysfunction. High RV MPI and LV MPI were detected in $9(30 \%)$ and $6(20 \%)$ patients, respectively.

Comparison between normal and high RV MPI cases regarding the disease and clinical characteristics as well as the laboratory parameters is demonstrated in Tables 4 \& 5. High RV MPI cases had significantly higher weight $(\mathrm{p}=0.015)$, BMI $(p=0.010)$ as well as longer mean duration of disease $(p=0.007)$ and treatment $(p=0.034)$ compared to those with low RV MPI. In addition, all cases with high RV MPI suffered from relapse $(p<0.001) ;$ a significantly higher percentage of them had steroid resistance $(p=0.025)$ and required immunesuppressive therapy $(p=0.025)$. All laboratory parameters did not differ significantly between the two groups ( $>00.05)$, except for platelet count which was significantly lower in patients with high RV MPI $(p=0.008)$ than those with low RV MPI. There were no significant differences between cases with high RV MPI and those with normal RV MPI as regard height, SBP, and DBP, hemoglobin, total leukocytic count, blood urea, serum creatinine, serum albumin, $\mathrm{A} / \mathrm{C}$ ratio, or blood cholesterol ( $\mathrm{p}>0.05)$.

Tables $6 \& 7$ show the differences between cases (normal and high LV MPI) as regards disease and clinical characteristics and laboratory parameters. The same significant differences between normal and high cases in RV were observed in the LV regarding the body weight $(\mathrm{p}=0.049)$, BMI $(\mathrm{p}=0.049)$, disease duration $(\mathrm{p}=0.031)$, treatment duration $(\mathrm{p}=0.026)$, relapse $(\mathrm{p}<0.001)$, steroid resistance $(p=0.003)$, immunesuppressive therapy $(\mathrm{p}=0.003)$, and platelet count $(\mathrm{p}=0.043)$.

There were 6 patients out of 30 found to have high MPI in both right and left ventricles and as shown in Table 8. These patients had significantly longer disease duration $(\mathrm{p}=0.031)$, treatment duration ( $\mathrm{p}$ $=0.026)$, and a higher percentage of them 
had steroid resistance $(\mathrm{p}=0.034)$ and administered immune-suppressive therapy $(\mathrm{p}=0.034)$. All 6 patients suffered from relapse. Table 9 shows that cases with relapse had significantly higher MPI $\mathrm{RV}$, MPI LV, weight, disease duration, treatment duration, and platelet count compared to cases with first attack.

Table 1: Demographic characteristics of both study groups

\begin{tabular}{|c|c|c|c|}
\hline Variable & PNS patients $(\mathrm{N}=30)$ & Controls $(\mathrm{N}=\mathbf{3 0})$ & P value \\
\hline \multicolumn{4}{|c|}{ Age (years) } \\
\hline Mean \pm SD & $6.5 \pm 3.3$ & $7.3 \pm 2.4$ & 0.334 \\
\hline \multicolumn{4}{|c|}{ Gender } \\
\hline Male, n (\%) & $19(63.3 \%)$ & $18(60 \%)$ & \multirow[t]{2}{*}{0.121} \\
\hline Female, n (\%) & $11(36.7 \%)$ & $97(40 \%)$ & \\
\hline
\end{tabular}

SD: standard deviation; $\mathrm{n}$ : number

Table 2: Comparison between cases \& controls regarding conventional echocardiography characteristics

\begin{tabular}{|l|c|c|c|}
\hline \multicolumn{1}{|c|}{ Variable } & Cases (N=30) & Controls (N=30) & P value \\
\hline PAP & \multicolumn{1}{c|}{ Conventional Ehocardiography (Mean \pm SD) } & 0.089 \\
\hline LA & $19.6 \pm 4.8$ & $17.9 \pm 2.1$ & 0.238 \\
\hline LVEDD & $2.3 \pm 0.2$ & $2.2 \pm 0.2$ & 0.100 \\
\hline LVESD & $3.7 \pm 0.5$ & $3.9 \pm 0.3$ & 0.134 \\
\hline LVPWD & $2.3 \pm 0.3$ & $2.4 \pm 0.3$ & 0.934 \\
\hline EF & $0.6 \pm 0.2$ & $0.6 \pm 0.1$ & 0.510 \\
\hline FS & $36.9 \pm 3.6$ & $37.4 \pm 2.5$ & 0.579 \\
\hline RVD & $67.2 \pm 4.5$ & $67.7 \pm 2.7$ & 0.061 \\
\hline
\end{tabular}

PAP: pulmonary artery pressure; LAD: left atrial diameter; LVEDD: left ventricular end diastolic diameter; LVESD: left ventricular end systolic diameter; LVPWD: left ventricular posterior wall diameter; EF: ejection fraction; FS: fraction shortening; RVD: RV dimension.

Table 3. Comparison between cases \& controls regarding tissue Doppler

\begin{tabular}{|c|c|c|c|}
\hline Variable & Cases $(\mathrm{N}=\mathbf{3 0})$ & Controls $(\mathrm{N}=30)$ & P value \\
\hline \multicolumn{4}{|c|}{ Tissue Doppler of right ventricle $($ Mean \pm SD) } \\
\hline IVRT & $54.8 \pm 12.2$ & $56.8 \pm 3.8$ & 0.408 \\
\hline IVCT & $43.1 \pm 6.1$ & $37.3 \pm 2.6$ & $<0.001 *$ \\
\hline ET & $270.4 \pm 15.3$ & $274.8 \pm 6.1$ & 0.151 \\
\hline $\mathbf{E}$ & $0.8 \pm 0.2$ & $0.9 \pm 0.2$ & 0.121 \\
\hline $\mathbf{A}$ & $0.4 \pm 0.1$ & $0.4 \pm 0.1$ & 0.700 \\
\hline $\mathbf{E} / \mathbf{A}$ & $1.8 \pm 0.3$ & $1.8 \pm 0.4$ & 0.991 \\
\hline MPI RV & $0.37 \pm 0.04$ & $0.34 \pm 0.02$ & 0.006* \\
\hline \multicolumn{4}{|l|}{ MPI RV, n (\%) } \\
\hline High & $9(30.0 \%)$ & $0(0.0 \%)$ & \multirow[t]{2}{*}{$0.002 *$} \\
\hline Normal & $21(70.0 \%)$ & $30(100.0 \%)$ & \\
\hline \multicolumn{4}{|c|}{ Tissue Doppler of left ventricle (Mean \pm SD) } \\
\hline IVRT & $57.9 \pm 6.5$ & $53.9 \pm 3.7$ & 0.005* \\
\hline IVCT & $43.1 \pm 8.4$ & $50.3 \pm 15.7$ & 0.032* \\
\hline ET & $275.3 \pm 11.4$ & $278.9 \pm 4.0$ & 0.111 \\
\hline $\mathbf{E}$ & $0.9 \pm 0.2$ & $1.0 \pm 0.2$ & $0.003^{*}$ \\
\hline $\mathbf{A}$ & $0.5 \pm 0.1$ & $0.6 \pm 0.1$ & $0.003^{*}$ \\
\hline $\mathbf{E} \backslash \mathbf{A}$ & $1.8 \pm 0.2$ & $1.8 \pm 0.2$ & 0.901 \\
\hline MPI LV & $0.37 \pm 0.04$ & $0.35 \pm 0.01$ & 0.009* \\
\hline \multicolumn{4}{|l|}{ MPI LV, n (\%) } \\
\hline High & $6(20.0 \%)$ & $0(0.0 \%)$ & \multirow[t]{2}{*}{ 0.024* } \\
\hline Normal & $24(80.0 \%)$ & $30(100.0 \%)$ & \\
\hline
\end{tabular}

IVRT: isovolumic relaxation time; IVCT: isovolumic contraction time; ET: ejection time; A: A wave; E: E wave; MPI RV: right ventricular myocardial performance index; MPI LV: left ventricular myocardial performance index;

Copyright 2021. All rights reserved (C) ESPNT ( geget ) 
Table 4: Comparison of clinical and disease characteristics between normal and high RV MPI cases

\begin{tabular}{|c|c|c|c|}
\hline Variable & High $(\mathrm{N}=9)$ & Normal $(\mathrm{N}=\mathbf{2 1})$ & $P$ value \\
\hline \multicolumn{4}{|c|}{ Clinical characteristics (Mean \pm SD) } \\
\hline Weight (kg) & $29.2 \pm 12.4$ & $20.4 \pm 6.3$ & $0.015 *$ \\
\hline Height (cm) & $120.2 \pm 18.0$ & $113.2 \pm 14.7$ & 0.274 \\
\hline BMI $\left(\mathrm{Kg} / \mathrm{m}^{2}\right)$ & $19.1 \pm 3.8$ & $15.7 \pm 2.7$ & $0.010 *$ \\
\hline SBP (mmHg) & $108.8 \pm 9.3$ & $102.1 \pm 9.6$ & 0.090 \\
\hline DBP (mmHg) & $68.2 \pm 10.7$ & $65.5 \pm 8.0$ & 0.444 \\
\hline \multicolumn{4}{|c|}{ Disease characteristics (Mean \pm SD) } \\
\hline Disease duration (months) & $26.4 \pm 8.8$ & $11.9 \pm 13.8$ & $0.007 *$ \\
\hline Treatment duration (months) & $22.4 \pm 5.3$ & $11.8 \pm 13.8$ & $0.034 *$ \\
\hline \multicolumn{4}{|c|}{ Attack, n (\%) } \\
\hline Relapse & $9(100.0 \%)$ & $5(23.8 \%)$ & \multirow[t]{2}{*}{$<0.001 *$} \\
\hline First attack & $0(0.0 \%)$ & $16(76.2 \%)$ & \\
\hline \multicolumn{4}{|c|}{ Steroid sensitivity, n (\%) } \\
\hline Sensitive & $7(77.8 \%)$ & $21(100.0 \%)$ & \multirow[t]{2}{*}{$0.025 *$} \\
\hline Resistance & $2(22.2 \%)$ & $0(0.0 \%)$ & \\
\hline \multicolumn{4}{|c|}{ Immuno-suppressive, n (\%) } \\
\hline Yes & $2(22.2 \%)$ & $0(0.0 \%)$ & \multirow[t]{2}{*}{ 0.025* } \\
\hline No & $7(77.8 \%)$ & $21(100.0 \%)$ & \\
\hline
\end{tabular}

BMI: body mass index; SBP: systolic blood pressure; DBP: diastolic blood pressure; SD: standard deviation;

* significant at $\mathrm{p} \leq 0.05$

Table 5: Comparison of laboratory parameters between normal and high RV MPI cases

\begin{tabular}{|l|c|c|c|}
\hline \multicolumn{1}{|c|}{ Lariable } & High (N=9) & Normal (N=21) & P value \\
\hline Hemoglobin & $11.8 \pm 0.8$ & $11.4 \pm 1.3$ & 0.406 \\
\hline White Blood Cells & $8.4 \pm 2.7$ & $8.4 \pm 3.4$ & 0.971 \\
\hline Platelet & $331 \pm 76.7$ & $468.1 \pm 185.3$ & $\mathbf{0 . 0 0 8 *}$ \\
\hline Blood Urea & $26.3 \pm 13.0$ & $32.4 \pm 18.5$ & 0.382 \\
\hline Serum Creatinine & $0.3 \pm 0.2$ & $0.4 \pm 0.2$ & 0.368 \\
\hline Serum Albumin & $1.8 \pm 0.4$ & $1.7 \pm 0.5$ & 0.608 \\
\hline Albumin/Creatinine ratio & $3.9 \pm 2.8$ & $5.9 \pm 4.5$ & 0.225 \\
\hline Cholesterol & $402.7 \pm 65.2$ & $424.6 \pm 99.7$ & 0.551 \\
\hline
\end{tabular}

Table 6: Comparison of clinical \& disease characteristics between normal \& high LV MPI cases.

\begin{tabular}{|c|c|c|c|}
\hline Variable & High $(\mathrm{N}=6)$ & Normal $(\mathbf{N}=24)$ & P value \\
\hline \multicolumn{4}{|c|}{ Clinical characteristics (Mean \pm SD) } \\
\hline Weight (kg) & $29.7 \pm 11$ & $21.4 \pm 8.3$ & 0.049* \\
\hline Height (cm) & $121.7 \pm 17.8$ & $113.8 \pm 15.2$ & 0.279 \\
\hline BMI $\left(\mathrm{Kg} / \mathbf{m}^{2}\right)$ & $19.2 \pm 3.0$ & $16.1 \pm 3.3$ & 0.049* \\
\hline SBP (mmHg) & $108.3 \pm 11.7$ & $103.1 \pm 9.3$ & 0.248 \\
\hline DBP (mmHg) & $68.3 \pm 11.7$ & $65.8 \pm 8.2$ & 0.537 \\
\hline \multicolumn{4}{|c|}{ Disease characteristics (Mean \pm SD) } \\
\hline Disease duration (months) & $25.7 \pm 9.4$ & $13.9 \pm 14.2$ & 0.031* \\
\hline Treatment duration (months) & $21.7 \pm 5.3$ & $13.3 \pm 13.7$ & 0.026* \\
\hline \multicolumn{4}{|c|}{ Attack n (\%) } \\
\hline Relapse & $6(100.0 \%)$ & $8(33.3 \%)$ & \multirow[t]{2}{*}{$<0.001 *$} \\
\hline First attack & $0(0.0 \%)$ & $16(66.7 \%)$ & \\
\hline \multicolumn{4}{|c|}{ Steroid sensitivity $\mathrm{n}(\%)$} \\
\hline Sensitive & $4(66.7 \%)$ & $24(100.0 \%)$ & \multirow[t]{2}{*}{ 0.003* } \\
\hline Resistance & $2(33.3 \%)$ & $0(0.0 \%)$ & \\
\hline \multicolumn{4}{|c|}{ Immuno-suppressive n (\%) } \\
\hline Yes & $2(33.3 \%)$ & $0(0.0 \%)$ & \multirow[t]{2}{*}{ 0.003* } \\
\hline No & $4(66.7 \%)$ & $24(100.0 \%)$ & \\
\hline
\end{tabular}

BMI: body mass index; SBP: systolic blood pressure; DBP: diastolic blood pressure; SD: standard deviation;

\section{Copyright 2021. All rights reserved (C) ESPNT ( geget )}


Table 7: Comparison of laboratory parameters between normal and high LV MPI cases.

\begin{tabular}{|l|c|c|c|}
\hline \multicolumn{1}{|c|}{ Variable } & High (N=6) & Normal (N=24) & P value \\
\hline Hemoglobin & \multicolumn{2}{c|}{ Laboratory parameters (Mean \pm SD) } & 0.462 \\
\hline White Blood Cells & $11.8 \pm 0.9$ & $11.4 \pm 1.2$ & 0.568 \\
\hline Platelet & $7.8 \pm 3.0$ & $8.6 \pm 3.2$ & $\mathbf{0 . 0 4 3 *}$ \\
\hline Blood Urea & $337.8 \pm 86.3$ & $449.3 \pm 181.3$ & 0.338 \\
\hline Serum Creatinine & $24.5 \pm 11.7$ & $32.1 \pm 18.0$ & 0.590 \\
\hline Serum Albumin & $0.3 \pm 0.2$ & $0.4 \pm 0.2$ & 0.322 \\
\hline Albumin/Creatinine ratio & $3.8 \pm 3.1$ & $5.6 \pm 4.3$ & 0.926 \\
\hline Cholesterol & $1.8 \pm 0.2$ & $1.8 \pm 0.5$ & 0.960 \\
\hline
\end{tabular}

Table 8: Comparison between patients with high \& normal MPI of both ventricles as regards disease characteristics

\begin{tabular}{|c|c|c|c|}
\hline Variable & $\operatorname{High}(\mathrm{N}=6)$ & Normal ( $\mathbf{N}=\mathbf{2 4})$ & P value \\
\hline Disease duration (months) (Mean \pm SD) & $25.7 \pm 9.4$ & $13.9 \pm 14.2$ & 0.031* \\
\hline Treatment duration (months)(Mean \pm SD) & $21.7 \pm 5.3$ & $13.3 \pm 13.7$ & $0.026 *$ \\
\hline \multicolumn{4}{|c|}{ Attack, n (\%) } \\
\hline Relapse & $6(100.0 \%)$ & $8(33.3 \%)$ & \multirow[t]{2}{*}{ 0.005* } \\
\hline First attack & $0(0.0 \%)$ & $16(66.7 \%)$ & \\
\hline \multicolumn{4}{|c|}{ Steroid sensitivity, n (\%) } \\
\hline Sensitive & $4(66.7 \%)$ & $24(100.0 \%)$ & \multirow[t]{2}{*}{$0.034 *$} \\
\hline Resistance & $2(33.3 \%)$ & $0(0.0 \%)$ & \\
\hline \multicolumn{4}{|c|}{ Immuno-suppressive, n (\%) } \\
\hline Yes & $2(33.3 \%)$ & $0(0.0 \%)$ & \multirow[t]{2}{*}{$0.034 *$} \\
\hline No & $4(66.7 \%)$ & $24(100.0 \%)$ & \\
\hline
\end{tabular}

$\mathrm{n}$ : number; $*$ significant at $\mathrm{p} \leq 0.05$

Table 9. Comparison between cases with first attack and those with relapse

\begin{tabular}{|c|c|c|c|c|c|}
\hline & & & & & P-value \\
\hline & Mean & SD & Mean & SD & \\
\hline PAP & 20.3 & 5.1 & 18.9 & 4.5 & 0.452 \\
\hline LA & 2.3 & 0.3 & 2.2 & 0.2 & 0.185 \\
\hline LVEDD & 3.9 & 0.5 & 3.6 & 0.4 & 0.098 \\
\hline LVESD & 2.3 & 0.4 & 2.2 & 0.3 & 0.832 \\
\hline LVPWD & 0.6 & 0.2 & 0.6 & 0.1 & 0.684 \\
\hline EF & 36.7 & 4.2 & 37 & 3.1 & 0.833 \\
\hline FS & 66.9 & 5.1 & 67.4 & 4 & 0.790 \\
\hline RVD & 1.3 & 0.1 & 1.2 & 0.1 & 0.166 \\
\hline IVRT & 56.1 & 17.2 & 53.7 & 5.5 & 0.623 \\
\hline IVCT & 45.6 & 7.6 & 41 & 3.5 & 0.053 \\
\hline ET & 269 & 17.1 & 271.6 & 13.9 & 0.647 \\
\hline MPI RV & 0.39 & 0.05 & 0.35 & 0.01 & 0.009* \\
\hline E wave & 0.8 & 0.2 & 0.8 & 0.1 & 0.744 \\
\hline A wave & 0.4 & 0.1 & 0.4 & 0.1 & 0.754 \\
\hline E/A Ratio & 1.8 & 0.2 & 1.8 & 0.3 & 0.604 \\
\hline IVRT & 60.6 & 6.7 & 55.6 & 5.6 & 0.036 \\
\hline IVCT & 42.5 & 12 & 43.6 & 3.3 & 0.730 \\
\hline ET & 271.4 & 15.4 & 278.8 & 4.3 & 0.107 \\
\hline MPI LV & 0.39 & 0.04 & 0.35 & 0.02 & $<0.001^{*}$ \\
\hline E wave & 0.9 & 0.2 & 0.9 & 0.2 & 0.800 \\
\hline A wave & 0.5 & 0.2 & 0.5 & 0.1 & 0.657 \\
\hline E/A Ratio & 1.7 & 0.3 & 1.8 & 0.2 & 0.493 \\
\hline
\end{tabular}

\section{Copyright 2021. All rights reserved $\odot$ ESPNT ( geget )}


Table 9. Comparison between cases with first attack and those with relapse (Continued)

\begin{tabular}{|c|c|c|c|c|c|}
\hline & \multicolumn{2}{|c|}{ Relapse } & \multicolumn{2}{|c|}{ First attack } & \multirow[t]{2}{*}{ P Value } \\
\hline & Mean & SD & Mean & SD & \\
\hline Weight (kg) & 27.1 & 11.1 & 19.4 & 5.6 & $0.030 *$ \\
\hline Height (cm) & 120.1 & 15 & 111.1 & 15.7 & 0.120 \\
\hline BMI $\left(\mathrm{Kg} / \mathrm{m}^{2}\right)$ & 18 & 3.8 & 15.7 & 2.7 & 0.060 \\
\hline SBP (mmHg) & 105.6 & 10.1 & 102.8 & 9.7 & 0.440 \\
\hline DBP (mmHg) & 67.4 & 9 & 65.3 & 8.8 & 0.521 \\
\hline Disease duration (months) & 53.9 & 55.1 & 7.3 & 9.8 & $0.008 *$ \\
\hline Treatment duration (months) & 53.1 & 55.5 & 7.1 & 9.9 & 0.009* \\
\hline Hemoglobin & 11.7 & 0.8 & 11.3 & 1.4 & 0.357 \\
\hline White blood Cells & 7.7 & 2.5 & 9 & 3.6 & 0.251 \\
\hline Platelets & 332.4 & 67.6 & 509.7 & 192.9 & 0.003* \\
\hline Blood Urea & 25 & 11.3 & 35.4 & 19.9 & 0.094 \\
\hline Serum Creatinine & 0.4 & 0.2 & 0.4 & 0.2 & 0.796 \\
\hline Serum Albumin & 1.8 & 0.4 & 1.7 & 0.5 & 0.374 \\
\hline Albumin/Creatinine ratio & 4.9 & 3.4 & 5.6 & 4.7 & 0.654 \\
\hline Cholesterol & 404.9 & 64 & 429.5 & 109 & 0.466 \\
\hline
\end{tabular}

SD: standard deviation; PAP: pulmonary artery pressure; LAD: left atrial diameter; LVEDD: left ventricular end diastolic diameter; LVESD: left ventricular end systolic diameter; LVPWD: left ventricular posterior wall diameter; EF: ejection fraction; FS: fraction shortening; RVD: RV dimension; IVRT: isovolumic relaxation time; IVCT: isovolumic contraction time; ET: ejection time; A: A wave; E: E wave; MPI RV: right ventricular myocardial performance index; MPI LV: left ventricular myocardial performance index; BMI: body mass index; SBP: systolic blood pressure; DBP: diastolic blood pressure; * significant at $\mathrm{p} \leq 0.05$.

\section{DISCUSSION}

The increased incidence of heart disease in patients with PNS has been reported by previous studies. Tissue Doppler imaging is a practical echocardiographic tool that possesses several advantages, being non-invasive, not time-consuming, relatively available, and software-independent compared to other diagnostic modalities [18]. Tissue Doppler imaging can be used to evaluate the systolic and diastolic myocardial motions, so it can be used to assess both systolic and diastolic dysfunctions [19]. The European Society of Cardiology and the American Society of Echocardiography recommend the use of tissue Doppler imaging for the evaluation of diastolic and systolic functions [20,21].

The aim of this study was to compare the findings of conventional echocardiography and tissue Doppler imaging in children with NS.
Echocardiography can demonstrate structural changes in the heart. In the present study, conventional echocardiography did not reveal any significant differences regarding the dimensions of right and left ventricles between PNS patients and healthy controls. These findings coincide with those of Saleh et al [22] and Abd Elaziz et al. [23] who found that there was no significant difference between patient and control group as regard these conventional echocardiographic parameters. However, other previous studies reported significant differences by echocardiography between PNS patients and controls. Du et al. [24] showed that pulmonary arterial pressure (PAP) Increased in children with nephrotic syndrome. Qin et al. [5] stated that patients with PNS had larger RV dimension and RVEDD by echocardiography compared to normal controls. 
In the present study, the parameters of conventional echocardiography indicated the absence of systolic or diastolic dysfunction of ventricles in our patients. However, tissue Doppler imaging of the right and left ventricles yielded significant differences between PNS patients and the healthy controls.

As regards the right ventricle, tissue Doppler echocardiography showed that isovolumetric contraction time (IVCT) was significantly prolonged in the patient group compared to the control group $(\mathrm{p}<$ 0.001 ). Similar results were reported by Saleh et al. [22] who found that IVCT was significantly increased in NS patients $(\mathrm{p}=$ 0.02). Prolonged IVCT indicates systolic dysfunction and occurs in cases of increased afterload as ventricular contraction is slower than in control subjects [25, 26]. Egan et al. [27] postulated that cardiac edema could also account for myocardial dysfunction, as it increases myocardial stiffness and induces contractile dysfunction. No other parameters of RV that were measured by tissue Doppler imaging showed significant differences between patients and control subjects, a finding which suggests the lack of RV diastolic dysfunction in our sample of patients.

The MPI evaluates the combined myocardial systolic and diastolic function [28] and was reported to be a more reliable parameter for the evaluation of systolic and diastolic function than the conventional echocardiographic indices [17] Calculation of RV MPI revealed that $30 \%$ of patients had an increase in MPI values, which are apparently caused by increased RV IVCT. Similar results were reported by Saleh et al. [22]. In contrast, Abd Elaziz et al.[23] showed that there was no significant difference between patient and control groups considering MPI.

As regards the left ventricle, tissue Doppler echocardiography revealed significant differences between cases and control groups, with the cases having prolonged IVRT, shortened IVCT as well as decreased $\mathrm{E}$ and $\mathrm{A}$ waves. LV MPI increased in $20 \%$ of nephrotic cases, apparently due to the increase in IVRT. These results were concordant with those of Saleh et al. [22] study. The IVRT was reported to be increased in both systolic and diastolic dysfunction [26].These findings indicated the presence of LV diastolic dysfunction in PNS patients. The ability of tissue Doppler imaging to detect early LV diastolic dysfunction was also reported by Lindblad et al. [29] in children with chronic kidney disease.

Considering the results of the current study, it appears that tissue Doppler imaging can detect early, occult systolic and diastolic ventricular dysfunctions before being detectable by conventional echocardiography. Early detection of ventricular dysfunction is pivotal to initiate early treatment and prevent progression of heart disease into heart failure.

The current study attempted to assess factors which are associated with increased MPI. There was a significant association between high MPI of the right or left ventricles and disease duration. Obviously, the disease and treatment durations were significantly more with relapse than with first attack. The MPI of both right and left ventricles were also significantly higher in relapse patients indicating a more severe degree of myocardial dysfunction in those patients. 
This agrees with the results of Qin et al. [5] who demonstrated that patients with increased RV dysfunction had a longer duration of disease $(p=0.015)$. On the other hand, Saleh et al. [22] stated that there were no significant differences between patients with RV dysfunction and those with normal RV function as regards the duration of illness.

Our study showed that there was an association between high MPI and steroid resistance $(\mathrm{p}=0.025)$, which could be due to long term administration of steroid and cytotoxic therapy. On the other hand, Qin et al.[5] found no differences in terms of steroid dependence or resistance.

The results of our study showed the lack of significant differences between cases with high RV or LV MPI and those with normal MPI as regards height, systolic blood pressure (SBP) and diastolic blood pressure (DBP). This is in line with Qin et al. [5] who stated that no significant difference in blood pressure was detected between patients with normal and increased RV peak pressure and RV end-diastolic pressure. On the other hand, Saleh et al. [22] stated that DBP was significantly increased $(\mathrm{p}=$ 0.002) in patients with RV diastolic dysfunction (high MPI) than those with normal RV diastolic function. Also, Bagga et al [30] found significant increase in DBP in patient group than control group, especially with steroid resistant NS. In addition, Hooman et al. [31] found

\section{CONCLUSION}

From the results of this study, we conclude that LV and RV dysfunction (high MPI) are not uncommon in children with PNS, occurring in $20 \%$ and $30 \%$ of NS cases, respectively. Both conditions that there was a statistically significant difference in SBP and DBP between patient and control groups.

In our study, both weight and BMI were significantly increased in cases with high MPI (in both left and right ventricles) than those with normal MPI. Similarly, the weight was significantly higher among patients with relapse compared to those with first attack. This could be due to long-term steroid therapy. This agrees with Rüth et al. [32] and Hooman et al. [31] as glucocorticoids induce an increase in BMI that is in proportion to the dose and duration of therapy. Also, in Lindblad et al. [29] study, BMI was independent predictor of LV diastolic dysfunction. However, Abd Elaziz et al. [23] found that there was no significant difference between NS patients and control group as regards BMI.

There was a significant increase in platelet count in our study in cases with high MPI of RT and LT ventricles than those with normal RT and LV MPI. As well, the platelet count was significantly higher among relapse cases than those with first attack. Our study agrees with Niaudet et al. [33] who stated that the increase in platelet count and platelet aggregability have been described in nephrotic patients. As we mentioned earlier in the introduction section, this increase in platelet count can play a role in the pathogenesis of heart disease in nephrotic patients.

are significantly associated with disease duration, treatment duration, relapse, steroid resistance, and administration of immune- suppressive drugs. 


\section{ABBREVIATIONS}

\begin{tabular}{|l|l|l|l|}
\hline A/C & Albumin /Creatinine & LVES & Left Ventricular End Systolic Diameter \\
DBP & Diastolic Blood Pressure & LVPW & Left Ventricular Posterior Wall \\
EF & Ejection Fraction & MPI & Myocardial Performance Index \\
ET & Ejection Time & NS & Nephrotic Syndrome \\
IVCT & Isovolumic Contraction Time & PAP & Pulmonary Arterial Pressure \\
IVRT & Isovolumic Relaxation Time & PNS & Primary Nephrotic Syndrome \\
LV & Left Ventricle & RV & Right Ventricle \\
LVED & Left Ventricular End Diastolic Diameter & SBP & Systolic Blood Pressure \\
\hline
\end{tabular}

\section{REFERENCES}

1. Banh TH, Hussain-Shamsy N, Patel V, Vasilevska-Ristovska J, Borges K, Sibbald C, Lipszyc D, Brooke J, Geary D, Langlois V, Reddon M, Pearl R, Levin L, Piekut M, Licht CP, Radhakrishnan S, Aitken-Menezes K, Harvey E, Hebert D, Piscione TD, Parekh RS. Ethnic Differences in Incidence and Outcomes of Childhood Nephrotic Syndrome. Clin J Am Soc Nephrol 2016; 11(10): 1760-8.

2. Chanchlani R, Parekh RS. Ethnic Differences in Childhood Nephrotic Syndrome. Front Pediatr 2016; 4(39).

3. Kerlin BA, Blatt NB, Fuh B, Zhao S, Lehman A, Blanchong C, Mahan JD, Smoyer WE. Epidemiology and risk factors for thromboembolic complications of childhood nephrotic syndrome: a Midwest Pediatric Nephrology Consortium (MWPNC) study. J Pediatr 2009; 155(1): 105-10, 10.e1.

4. Dumas De La Roque C, Prezelin-Reydit M, Vermorel A, Lepreux $\mathrm{S}$, Deminière $\mathrm{C}$, Combe C, Rigothier C. Idiopathic Nephrotic Syndrome: Characteristics and Identification of Prognostic Factors. J Clin Med 2018; 7(9).

5. Qin Q, Xu R, Dong J, Xia W, Sun R. Evaluation of right ventricle function in children with primary nephrotic syndrome. Pediatr Neonatol 2010; 51(3): 166-71.

6. Moreira-Rodrigues $M$, Roncon-Albuquerque R, Jr., Henriques-Coelho T, Lourenço AP, Sampaio-Maia B, Santos J, Pestana M, LeiteMoreira AF. Cardiac remodeling and dysfunction in nephrotic syndrome. Kidney Int 2007; 71(12): 1240-8.

7. Nakamura A, Niimi R, Kurosaki K, Yanagawa $Y$. Factors influencing cardiovascular risk following termination of glucocorticoid therapy for nephrotic syndrome. Clin Exp Nephrol 2010; 14(5): 457-62.

8. Ozkan G, Adar A, Ulusoy S, Bektaş H, Kırış A, Fidan M, Celik S. Presence of fragmented QRS and its correlation with myocardial performance index in patients with nephrotic syndrome. Anadolu Kardiyol Derg 2014; 14(5): 450-5.

9. Correia Pinto J, Henriques-Coelho $\mathrm{T}$, Roncon-Albuquerque R, Jr., Leite-Moreira AF. Differential right and left ventricular diastolic tolerance to acute afterload and NCX gene expression in Wistar rats. Physiol Res 2006; 55(5): 513-26.

10. Nalcacioglu H, Ozkaya O, Kafali HC, Tekcan D, Baysal K. Cardiac Functions in Children with Steroid Sensitive Nephrotic Syndrome. Pediatr Int 2020; n/a(n/a).

11. Loscalzo J. Venous Thrombosis in the Nephrotic Syndrome. 2013; 368(10): 9568.

12. Suri D, Ahluwalia J, Saxena AK, Sodhi KS, Singh P, Mittal BR, Das R, Rawat A, Singh S. Thromboembolic complications in childhood nephrotic syndrome: a clinical profile. Clin Exp Nephrol 2014; 18(5): 80313.

13. Hari P, Khandelwal P, Smoyer WE. Dyslipidemia and cardiovascular health in

Copyright 2021. All rights reserved $\subset$ ESPNT ( geget ) 
childhood nephrotic syndrome. Pediatr Nephrol 2020; 35(9): 1601-19.

14. Vaziri ND. Disorders of lipid metabolism in nephrotic syndrome: mechanisms and consequences. Kidney Int 2016; 90(1): 4152.

15. Sadeghpour A, Alizadehasl A. Chapter 5 Echocardiography. In: Maleki M, Alizadehasl A, Haghjoo M, editors. Practical Cardiology: Elsevier; 2018. p. 67111.

16. Eidem BW, McMahon CJ, Cohen RR, Wu J, Finkelshteyn I, Kovalchin JP, Ayres NA, Bezold LI, O'Brian Smith E, Pignatelli RH. Impact of cardiac growth on Doppler tissue imaging velocities: a study in healthy children. J Am Soc Echocardiogr 2004; 17(3): 212-21.

17. Lakoumentas JA, Panou FK, Kotseroglou VK, Aggeli KI, Harbis PK. The Tei index of myocardial performance: applications in cardiology. Hellenic J Cardiol 2005; 46(1): 52-8

18. Draz IH, Mostafa FEZ, AbdelMassih AF, El-Sayed D, El-Kaffas R. The use of tissue Doppler imaging in the assessment of diastolic dysfunction in children with chronic lung diseases. Egypt Pediatr Assoc Gaz 2020; 68(1): 15.

19. Tissot C, Muehlethaler V, Sekarski N. Basics of Functional Echocardiography in Children and Neonates. Front Pediatr 2017; 5(235).

20. Nagueh SF, Appleton CP, Gillebert TC, Marino PN, Oh JK, Smiseth OA, Waggoner AD, Flachskampf FA, Pellikka PA, Evangelista A. Recommendations for the Evaluation of Left Ventricular Diastolic Function by Echocardiography. J Am Soc Echocardiogr 2009; 22(2): 107-33.

21. McMurray JJ, Adamopoulos S, Anker SD, Auricchio A, Böhm M, Dickstein K, Falk V, Filippatos G, Fonseca C, Gomez-Sanchez
MA, Jaarsma T, Køber L, Lip GY, Maggioni AP, Parkhomenko A, Pieske BM, Popescu BA, Rønnevik PK, Rutten FH, Schwitter J, Seferovic P, Stepinska J, Trindade PT, Voors AA, Zannad F, Zeiher A. ESC Guidelines for the diagnosis and treatment of acute and chronic heart failure 2012: The Task Force for the Diagnosis and Treatment of Acute and Chronic Heart Failure 2012 of the European Society of Cardiology. Developed in collaboration with the Heart Failure Association (HFA) of the ESC. Eur Heart J 2012; 33(14): 1787-847.

22. Saleh S, Elmaghraby K, Abdelfadil A, Mohamed H. Myocardial Performance Index in Nephrotic Syndrome. J Clin Exp Cardiolog 2018; 9(4): 585

23. AbdElaziz L, Abouzeid $H$, Fares $K$. Myocardial dysfunction in children with nephrotic syndrome, a tissue Doppler study. Zagazig University Medical Journal 2019; 25(3): 409-18.

24. Du ZD, Cao L, Liang L, Chen D, Li ZZ. Increased pulmonary arterial pressure in children with nephrotic syndrome. Arch Dis Child 2004; 89(9): 866-70.

25. Moreno R, Zamorano J, Almería C, PérezGonzález JA, Mataix L, Rodrigo JL, Herrera D, Aubele A, Pérez de Isla L, De Marco E, Sánchez-Harguindey L, Macaya C. Isovolumic Contraction Time by PulsedWave Doppler Tissue Imaging in Aortic Stenosis. Eur J Echocardiogr 2003; 4(4): 279-85.

26. Tei C, Dujardin KS, Hodge DO, Bailey KR, McGoon MD, Tajik AJ, Seward SB. Doppler echocardiographic index for assessment of global right ventricular function. J Am Soc Echocardiogr 1996; 9(6): 838-47.

27. Egan JR, Butler TL, Au CG, Tan YM, North $\mathrm{KN}$, Winlaw DS. Myocardial water handling and the role of aquaporins.

Copyright 2021. All rights reserved (C) ESPNT ( geget ) 
Biochim Biophys Acta 2006; 1758(8): 1043-52.

28. Tei C, Ling LH, Hodge DO, Bailey KR, Oh JK, Rodeheffer RJ, Tajik AJ, Seward JB. New index of combined systolic and diastolic myocardial performance: a simple and reproducible measure of cardiac function--a study in normals and dilated cardiomyopathy. J Cardiol 1995; 26(6): 357 66.

29. Lindblad YT, Axelsson J, Balzano R, Vavilis G, Chromek M, Celsi G, Bárány P. Left ventricular diastolic dysfunction by tissue Doppler echocardiography in pediatric chronic kidney disease. Pediatr Nephrol 2013; 28(10): 2003-13.

30. Bagga A, Ali U, Banerjee S, Kanitkar M, Phadke KD, Senguttuvan P, Sethi S, Shah M. Management of steroid sensitive nephrotic syndrome: revised guidelines. Indian Pediatr 2008; 45(3): 203-14.

31. Hooman N, Isa-Tafreshi $R$, Otukesh $H$, Mostafavi SH, Hallaji F. Carotid artery function in children with idiopathic nephrotic syndrome. Nefrologia 2013; 33(5): 650-6.

32. Rüth EM, Kemper MJ, Leumann EP, Laube GF, Neuhaus TJ. Children with steroidsensitive nephrotic syndrome come of age: long-term outcome. J Pediatr 2005; 147(2): 202-7.

33. Niaudet P, Boyer O. Idiopathic nephrotic syndrome in children: clinical aspects. In: Avner E, Harmon W, Niaudet P, Yoshikawa $\mathrm{N}$, Emma F, Goldstein S, editors. Pediatric nephrology. 7th ed. Berlin Springer; 2016. p. 850-93.

\section{Authors' contributions}

The submitted manuscript is the work of the author \& co-author.

All authors have contributed to authorship, have read and approved the manuscript. Conception and design of study: all authors Acquisition of data: $2^{\text {nd }}$ author.

Analysis and/or interpretation of data: $1^{\text {st }}$ and last author.

Drafting the manuscript: $1^{\text {st }}$ author.

Revising the manuscript critically for important intellectual content: $1^{\text {st }}$ and $2^{\text {nd }}$ author.

Approval of the version of the manuscript to be published: All authors.

\section{STATEMENTS}

\section{Consent for publication}

The attached manuscript its contents and materials have not been previously reported at any length or being considered for publishing elsewhere.

\section{Ethics approval and consent to participate}

The local ethical committee permitted the study under the Helsinki declaration of Bioethics and its later amendments. Informed consent (written form) was obtained from all participants or their caregivers.

\section{Availability of data}

The authors have indicated that the data and material are factual and genuine.

\section{Conflict of interest}

The authors have indicated they have no potential conflicts of interest to disclose.

\section{Funding}

The authors have indicated they have no financial relationships relevant to this article to disclose.

\section{Acknowledgments}

None.

Submitted:

$08 / 10 / 2021$

Accepted:

$16 / 12 / 2021$

Published Online: 31/12/2021

Copyright 2021. All rights reserved @ ESPNT ( geget ) 